\title{
Formulation and Evaluation of Nanoscale Solid Lipid Particles Containing a Hydrophilic Drug-Rasagiline Mesylate
}

\author{
Viveksarathi Kunasekaran, Kannan Krishnamoorthy* \\ Department of Pharmacy, Faculty of Engineering and Technology, Annamalai University, Annamalai Nagar-608 002, Tamil Nadu, India.
}

\begin{tabular}{l} 
ARTICLE INFO \\
\hline Article history: \\
Received on: $16 / 12 / 2015$ \\
Revised on: 05/02/2016 \\
Accepted on: 22/02/2016 \\
Available online: 28/05/2016 \\
\hline Key words: \\
Parkinson disease, Rasagiline \\
mesylate, Solid lipid \\
nanoparticles, Scanning \\
electron microscopy, \\
Transmission electron \\
microscopy.
\end{tabular}

\begin{abstract}
The present study was to prepare and characterize Rasagiline mesylate loaded nanoscale solid lipid particles and evaluate its In-vitro release. Rasagiline mesylate loaded solid lipid nanoparticles was fabricated by microemulsion technique and then characterized with respect to particle size, polydispersity index and zeta potential were measured by photon correlation spectroscopy. Morphological examination was visualized by transmission electron microscopy. The release of Rasagiline mesylate from solid lipid nanoparticles was performed by using the incubator shaking technique. The fabricated formulations particle size ranged from $160.20 \pm 3.2$ to $210.12 \pm 5.3 \mathrm{~nm}$ and the zeta potential measurements indicates a narrow size distribution. The Polydispersity index values shown narrow that means the nanoparticles are homogenous in nature. Drug loading and entrapment efficiency of RMSLN-I \& RMSLN-II were $33.34 \pm 1.45 \%$ \& $76.24 \pm 1.2 \%$ and $16.67 \pm 0.86 \%$ \& $73.75 \pm 1.02 \%$, respectively. TEM revealed the particles were monodisperse, uniform size and quasispherical shape. Release of Rasagiline mesylate loaded nanoscale solid lipid particles were shown efficient prolonged drug release and followed by Fickian diffusion mechanism. Furthermore, RM loaded SLNs were found to be stable, after 6 months of storage at different conditions. The developed solid lipid nanoparticles were able to control the drug release for a prolonged period of time.
\end{abstract}

\section{INTRODUCTION}

Rasagiline (N-propargyl-1 (R)-aminoindan) mesylate $(\mathrm{RM})$ is a novel second generation of propargylamine, selective, irreversible monoamine oxidase B (MAO-B) inhibitor used for the treatment of Parkinson's disease (PD) (Viveksarathi and Kannan, 2014). MAO is a flavin-containing enzyme located in outer mitochondrial membrane proteins in both peripheral organs and neuronal cells (Kersemans et al., 2013). The enzyme regulates the metabolic degradation of catecholamines and serotonin, and is found in two isoforms namely A and B. MAO$\mathrm{A}$ is involved in the metabolism of serotonin, noradrenaline and

\footnotetext{
* Corresponding Author

Kannan Krishnamoorthy, Assistant Professor, Department of Pharmacy, Faculty of Engineering and Technology, Annamalai University, Annamalai Nagar-608 002, Tamil Nadu, India.

Email:egkkannan@yahoo.co.in
}

dopamine whereas MAO-B metabolizes the dopamine neurotransmitter (Lakshmi Nayak and Claire Henchcliffe, 2008). The loss of melanin containing dopaminergic neurons in the substantia nigra (pars compacta) is associated with Parkinson disorder (Marcos Fernandez et al., 2011).

$\mathrm{PD}$ is recognized as the second most common, progressive neurodegenerative disorder in the world after Alzheimer's and it manifested clinically by motor symptoms, including rigidity, resting tremor and bradykinesia in the limbs followed by postural instability (Moussa BH Youdimaa and Marta Weinstock, 2002; Andreas-Antonios, 2013). The treatment of PD is restricted by an insufficiency in delivering therapeutic drugs into the brain relating to highly limited transport of compounds across the blood-brain barrier (Jack J Chen and David M Swope, 2007). A low molecular mass 400-500 Da and high lipophilic drugs can only cross the blood brain barrier (Mori et al., 1995). 
To overcome this obstruction, a variety of colloidal carriers have been implemented like liposomes (Mori et al., 1995), polymeric nanoparticles (Kreuter et al., 2003), solid lipid nanoparticles (Shuting et al., 2010) and dendrimers (Wolfgang Mehnert and Karsten Mad, 2001). Among these solid lipid nanoparticles become an important area of research in the field of drug delivery into the brain in a sustained period of time. The preparation of solid lipid nanoparticle (SLNs) techniques includes high pressure homogenizer, solvent emulsification, solvent diffusion, solvent evaporation, solvent injection, hot/cold homogenization, microemulsion, emulsification-diffusion, double emulsion, ultrasonication, spray drying and solvent supercritical fluid ( Viveksarathi and Kannan, 2015;Wolfgang Mehnert and Karsten Mad, 2001). However, we have chosen microemulsion technique, which is thermodynamically stable and most expedient for the preparation of solid lipid nanoparticles. Stearic acid is used as lipid solid, which is an endogenous saturated fatty acid found in an animal and plant sources, providing better biocompatibility and low toxicity (Patricia Severino et al., 2013; Attama et al., 2006). The melting point of stearic acid is higher than body temperature. So, it can be compatible with human tissues and neutral with respect to physiological fluids (Fundaro et al., 2000).

The objective of this study was to prepare the Rasagiline mesylate loaded solid lipid nanoparticles using stearic acid, polyethylene-polypropylene glycol and polysorbate 80 and to evaluate the In-vitro drug release and also predict the stability of the formulation in different storage conditions $25^{\circ} \mathrm{C} \pm 2{ }^{\circ} \mathrm{C} / 60 \% \mathrm{RH} \pm 5 \% \mathrm{RH}$ (Long term), $30^{\circ} \mathrm{C} \pm 2^{\circ} \mathrm{C} / 65 \% \mathrm{RH} \pm 5 \% \mathrm{RH}$ (Intermediate) and $40^{\circ} \mathrm{C} \pm 2^{\circ} \mathrm{C} / 75 \% \mathrm{RH} \pm 5 \% \mathrm{RH}$ (Accelerated condition), which can be assessed by particle size, polydispersity index, zeta potential entrapment efficiency and drug content (Viveksarathi et al., 2012; Viveksarathi and Kannan, 2013; Surajit Das et al., 2011).

\section{MATERIALS AND METHODS}

\section{Materials}

Rasagiline mesylate was a generous gift from Orchid health care Pvt Ltd (Chennai, India). Stearic acid and Tween 80 were obtained from SD Fine Chem Ltd (Mumbai, India). HPLC grade Acetonitrile and Lutrol were purchased from Sigma Aldrich (Bangalore, India) and Signet Chemical Lab (Mumbai, India), respectively. The analytical grade chemicals and reagents were used for all the experiments. Double distilled water was used after filtration through a $0.45 \mu \mathrm{m}$ membrane (cellulose acetate).

\section{Fabrication of Rasagiline-loaded SLNs}

The nanoscale solid lipid particles which contain the drug Rasagiline mesylate was fabricated from oil-in-water microemulsion technique as previously described by kannan et al., 2015 and Doijad et al., 2008. In this method, lipid phase containing stearic acid was melted at $69-70^{\circ} \mathrm{C}$ and the drug was dissolved in the melted lipid. Then the lipid phase was added drop wise into the aqueous phase containing Polaxamer-407 as surfactant and Polysorbate- 80 as a co-surfactant, which heated at the same temperature. A transparent, thermodynamically stable $\mathrm{o} / \mathrm{w}$ microemulsion was obtained under magnetic stirrer (Remi, India) at $500 \mathrm{rpm}$ for $15 \mathrm{~min}$. This resulting $\mathrm{o} / \mathrm{w}$ microemulsion was dispersed into cold aqueous medium under probe sonicator for 20 min (Lark, India) to solidify the nanoparticles in a volume ratio of 1:1 hot microemulsion to cold water. The fabricated Rasagiline mesylate loaded nanoscale solid lipid particles were freeze dried on a lyophilizer (lark, India.) at $-40^{\circ} \mathrm{C}$ temperature and operating pressure 0.4 bar. The dried powder was stored in a desiccator (Viveksarathi and Kannan, 2015a; Viveksarathi and Kannan, 2015b; Doijad et al., 2008). The Rasagiline mesylate loaded Nanoscale solid lipid particles was prepared using different ratio of drug to stearic acid. The ratio of drug to stearic acid (w/w) in RMSLNs I and RMSLNs II was 1:2 and 1:4, respectively.

\section{Determination of process yield}

The Rasagiline mesylate loaded nanoscale solid lipid particles process yield was determined using the following equation, process yield $=($ Practical yield/theoretical yield $) \times 100$ (Viveksarathi and Kannan, 2015a, Barnabas Wilson et al., 2008).

\section{Particle size distribution and Polydispersity index}

Particle size distribution and Polydispersity index of fabricated nanoscale solid lipid particles was measured by dynamic light scattering (DLS) or photon correlation spectroscopy (PCS) or quasi-elastic light scattering (QELS) at a scattering angle of $90^{\circ}$ using a Malvern Mastersizer 2000MS (Malvern Instruments Inc, Worcestershire, UK) and laser diffraction with a beam length of $2.40 \mathrm{~mm}$, range lens of $300 \mathrm{RF} \mathrm{mm}$, at $10.14 \%$ obscuration. Polydispersity index was calculated from Gaussian distribution which indicated the width of a particle size of distribution by using the equation: $\mathrm{D}(0.9)-\mathrm{D}(0.1) / \mathrm{D}(0.5)$.

Where, $\mathrm{D}(0.9), \mathrm{D}(0.5)$ and $\mathrm{D}(0.1)$ are correspondence to particle size immediately above $90 \%, 50 \%$ and $10 \%$ of the sample. The Malvern Mastersizer particle size measurements range between $0.02 \mu \mathrm{m}$ and $2000 \mu \mathrm{m}$ (Viveksarathi and Kannan, 2015b).

\section{Zeta potential measurements}

The surface charge and electrostatic stabilization of Rasagiline mesylate loaded nanoscale solid lipid particles were quantified by laser Doppler electrophoresis using Malvern Zetasizer Nano ZS (Malvern Instruments, UK). The sample was diluted with deionized distilled water with a conductivity adjusted to $50 \mu \mathrm{S} / \mathrm{cm}$ with $0.9 \%$ sodium chloride. The quantified electrophoretic mobility was converted to zeta potential by applying Helmholtz-Smoluchowski equation.

\section{Determination of Entrapment Efficiency}

The Rasagiline mesylate loaded nanoscale solid lipid particles entrapment efficiency (EE) was determined by analyzing the free drug content in the supernatant obtained after centrifuging at $16,000 \mathrm{rpm}$ for $30 \mathrm{mins}$ at $0^{\circ} \mathrm{C}$ (Remi C 24, Mumbai, India). Quantitative determination of RM-SLNs was conducted by HPLC 
(Thermoscientific, spectra system P-4000, USA) as previously described by Kannan et al., 2015 (22) and Marcos Fernandez et al., 2011 (Marcos Fernandez et al., 2014), using a UV detector (Kromosil 100) and $\mathrm{C}_{18}$ column (particle size $5 \mu \mathrm{m}$, $250 \mathrm{~mm} \times 4 \mathrm{~mm}$ ). The amount of entrapped drug was calculated as the total amount of drug minus free drug by using the following equation

Entrapment efficiency (\%) $=\frac{(\mathrm{W} 1 \text { (assay) }-\mathrm{W} 2)}{\mathrm{W} 1} \times 100$

Where, W1 -the initial amount of drug used in the formulation, W2 -amount of drug detected in the supernatant

\section{Determination of drug loading capacity}

Drug loading was determined by measuring the weight of drug in nanopartiles versus total weight of SLNs by using following equation (Guihua Huang et al., 2008):

Drug loading $(\%)=\frac{\text { Weight of drug in SLNs }}{\text { Total weight of SLNs }} \times 100$

\section{Determination of drug content}

Weighed amount of Rasagiline mesylate loaded solid lipid nanoparticles was diluted with mixture of acetonitrile:water $(5: 95, \mathrm{v} / \mathrm{v})$ and analyzed by HPLC method (Thermoscientific, spectra system P-4000, USA) using UV detector (Kromosil 100) and C18 column (particle size $5 \mu \mathrm{m}, 250 \mathrm{~mm} \times 4 \mathrm{~mm}$ ). The detection of wavelength was $265 \mathrm{~nm}$. Under these conditions the retention time of Rasagiline mesylate was at $4.627 \mathrm{~min}$ and concentration was calculated by relative to a calibration curve (Viveksarathi and Kannan, 2015c).

\section{Scanning electron microscopy}

The RM-SLNs was visualized using Scanning electron microscopy (SEM) Jeol JX 840-A (Jeol LTD, Tokyo, Japan) for morphological examination. Freeze dried samples mounted on aluminium stubs and then coated with gold using a sputter coater. The specimens were scanned at $15 \mathrm{kv}$ accelerated voltage.

\section{Transmission electron microscopy}

Transmission Electron Microscopy (TEM) is working based on transmitted electron and it has much higher resolution than SEM. Therefore TEM also be used to determine the sample surface morphology and crystallography. The Surface morphology of RM loaded nanoscale solid lipid particles were viewed under Transmission Electron Microscopy using JEOL JEM-2000 EXII TEM (Tokyo, Japan). The samples were negatively stained with $0.5 \%(\mathrm{w} / \mathrm{v})$ phosphotungstic acid solution and fixing on coated copper grids with carbon film and dried under vacuum pressure. The samples were diluted to $5 \mathrm{ml}$ in buffer to obtain a clear solution and scanned in all zones before the images are taken.

\section{In-vitro drug release studies}

In-vitro release study of Rasagiline mesylate loaded nanoscale solid lipid particles was determined by using dialysis bag method. Accurately weighed quantities of RM-SLNs were placed in the dialysis bag (cut-off $5 \mathrm{kDa}$, Himedia, India) and both ends were tightly sealed. The dialysis bag was positioned in a Beaker containing $100 \mathrm{ml}$ phosphate buffer at $\mathrm{pH}$ 7.4, which was magnetically stirred at $100 \mathrm{rpm}$ (Remi, India) and maintained at $37^{\circ} \mathrm{C} \pm 0.5^{\circ} \mathrm{C}$. At schedule time intervals, the $1 \mathrm{ml}$ of the release medium was withdrawn using micropipette and replaced with the same volume of fresh PBS. The samples were immediately filtered through a $0.45 \mu \mathrm{m}$ membrane filter (Elix, Mill-Q) and the content of Rasagiline mesylate was estimated after suitable dilution with a Thermoscientific HPLC (Spectra system P-4000, USA) with UV detector (Kromosil 100) and $\mathrm{C}_{18}$ column (Particle size $5 \mu \mathrm{m}, 250$ $\mathrm{mm} \times 4 \mathrm{~mm}$ ) at $265 \mathrm{~nm}$ (Barnabas Wilson et al., 2008; Shengpeng Wang et al., 2012).

\section{Drug releases kinetics}

The release of drug loaded nanoscale solid lipid particle statistics was studied using, zero-order, first-order, Higuchi and Korsmeyer-Peppas equation models (Viveksarathi and Kannan, 2012).

\section{Stability studies}

The stability study provides the evidence of fabricated nanoscale solid lipid particles quality varies with time under different environmental factors includes temperature, humidity and light. The study was carried out according to the international conference on harmonisation (ICH-QIA (R2) guidelines, 2003), with Rasagiline mesylate loaded nanoscale solid lipid particles. The samples were studies were performed over a period of 6 months at $25^{\circ} \mathrm{C} \pm 2{ }^{\circ} \mathrm{C} / 60 \% \mathrm{RH} \pm 5 \% \mathrm{RH}$ (Long term), $30^{\circ} \mathrm{C} \pm 2{ }^{\circ} \mathrm{C} / 65 \% \mathrm{RH} \pm 5 \% \mathrm{RH} \quad$ (Intermediate) and $40^{\circ} \mathrm{C} \pm 2{ }^{\circ} \mathrm{C} / 75 \% \mathrm{RH} \pm 5 \% \mathrm{RH}$ (Accelerated condition). The samples were evaluated at 0,3 and 6 months for their particle size, polydispersity index, zeta potential, entrapment efficiency, drug content and release rate (Viveksarathi and Kannan, 2012; Viveksarathi and Kannan, 2013; Surajit Das et al., 2011), In addition, samples were visually examined for any physical instability (separation and aggregation).

\section{RESULTS}

The percentage process yield of $86.21 \%$ and $84.72 \%$ were obtained from RMSLN-I and RMSLN-II, respectively (Table 1). Most critical of any nanoscale solid lipid particles fabrication strategy is the particle size distribution, which plays a major role in their adhesion to the cell membrane and interaction with the biological cells. Fig. $1 \& 2$. Shows the particle size distribution pattern of the Rasagiline mesylate loaded solid lipid particles and the mean particle size range was from $160.12 \pm 1.2 \mathrm{~nm}$ to $210.45 \pm 5.3 \mathrm{~nm}$ (Table 1). The size of nanoscale solid lipid particles are mainly depends on the factors like rate of addition and rate of stirring. The results acquired from dynamic light scattering are confirmed by scanning electron microscopy (SEM) or transmission electron microscopy (TEM). 


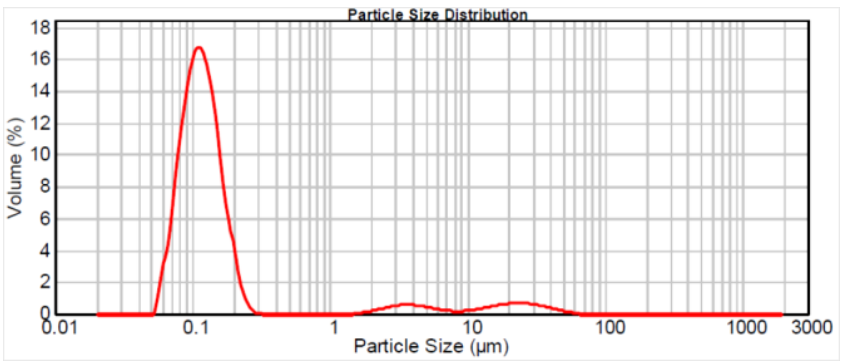

Fig. 1: Particle size distribution of RMSLN-I

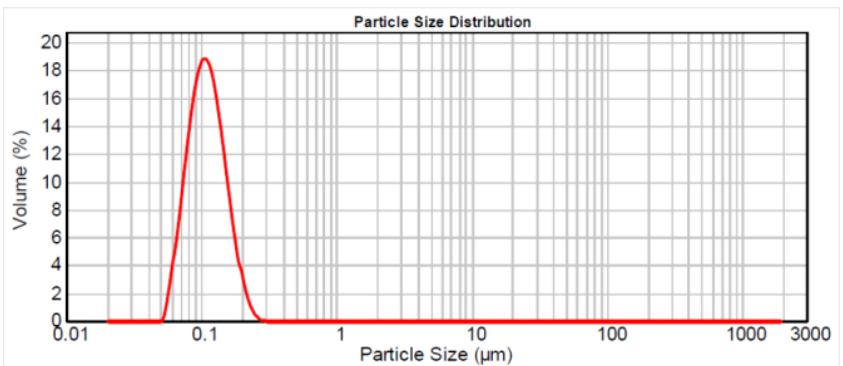

Fig. 2: Particle size distribution of RMSLN-II

The PdI was calculated on the basis of volumetric distribution of particles and provides the statistics about the homogeneity of particle size distribution. The formulated RMSLNs I \& II particle size distribution is unimodal and having a narrow range of 0.304 to 0.231 (Table 1).

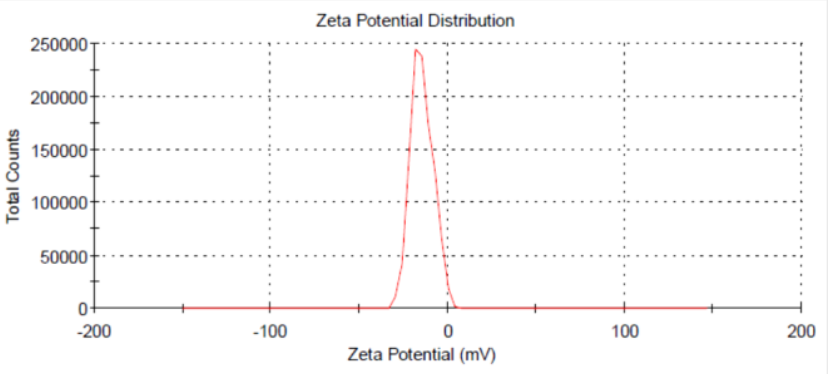

Fig. 3: Zeta potential measurements of RMSLN I

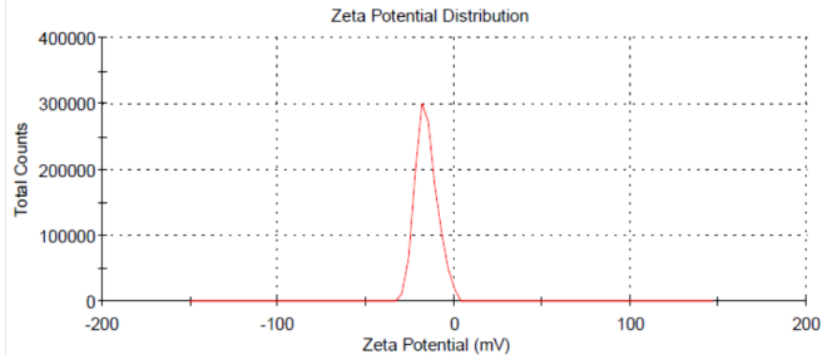

Fig. 4: Zeta potential measurements of RMSLN II.

Zeta potential is used to me-asure the surface charge of particles and electrostatic stabilization, through the electrostatic repulsion between particles (Fig. $3 \& 4$ ).

The higher positive or negative zeta potential value results, minimize the aggregation/flocculation of particles. The formulations RMSLN-I and RMSLN-II zeta potential value was -
36.7 \pm 6.10 and $-31.5 \pm 1.9 \mathrm{mV}$, respectively (Table 1 ). Drug loading and entrapment efficiency of RMSLN-I and RMSLN-II were $33.34 \pm 1.45 \%$ \& $76.24 \pm 1.2 \% \quad(n=3)$ and $16.67 \pm 0.86 \%$ \& $73.75 \pm 1.02 \%(n=3)$, respectively (Table 1$)$. Drug content of the formulation RMSLN-I and RMSLN-II was $96.63 \pm 0.42 \%$ and $94.17 \pm 1.12 \%$, respectively. Fig. 5: (a) \& (b) shows the results of scanning electron microscope, morphology was found to spherical shape.

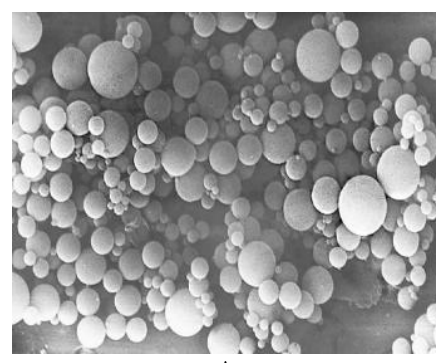

A

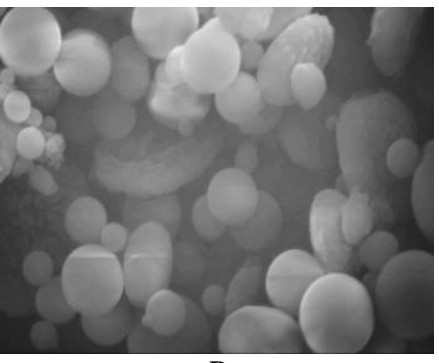

B
Fig. 5: (a). Scanning electron microscopy micrograph of RMSLN-I, (b). Scanning electron microscopy micrograph of RMSLN-II.

RMSLN-I and RMSLN-II Transmission electron microscope studies indicated the crystalline morphology of Rasagiline mesylate loaded nanoscale solid lipid particle was almost uniform size with spherical shape and few small particles aggregate into secondary particles because their very small dimension and high surface energy. Fig. 6 \& 7: Section (b) Selected area electron diffraction pattern shows the formulated RM-SLNs particles are polycrystalline structure.

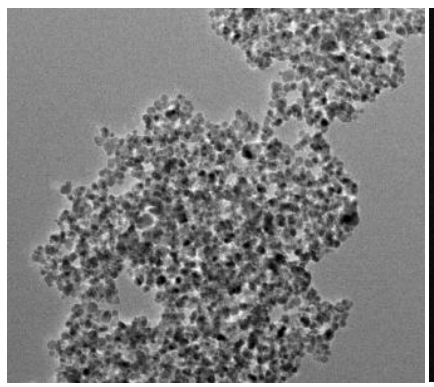

A

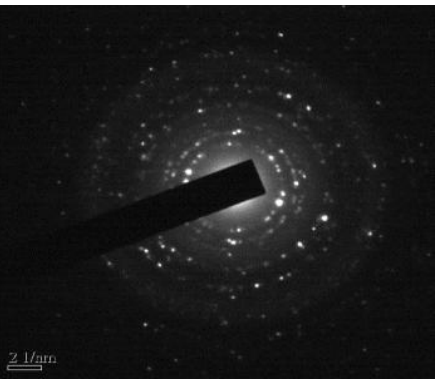

B
Fig. 6: (a) Transmission electron microscopy micrograph of RMSLN I and (b) the selected area electron diffraction pattern of RMSLN I.

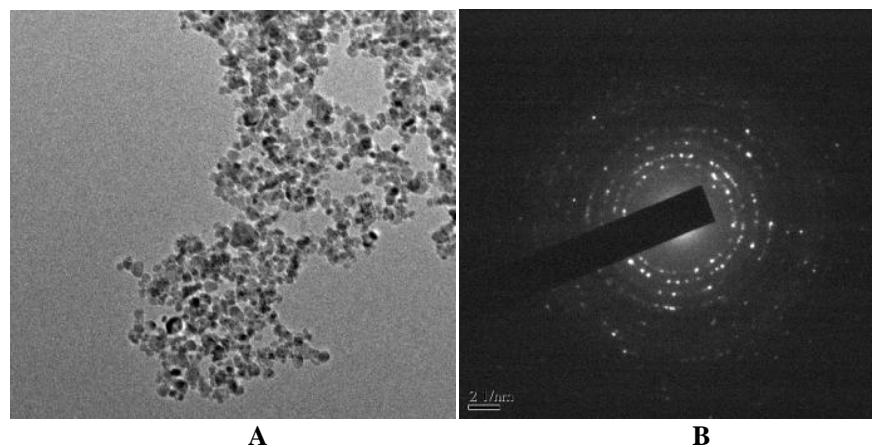

Fig. 7: (a) Transmission electron microscopy micrograph and (b) the selected area electron diffraction pattern of RMSLN II. 
Table 1: Characterization of RM-loaded solid lipid nanoparticles.

\begin{tabular}{lcc}
\hline \multirow{2}{*}{ Evaluation parameters } & \multicolumn{2}{c}{ Formulations code } \\
\cline { 2 - 3 } & RMSLN-I & RMSLN-II \\
\hline Process yield (\%) & 86.21 & 84.72 \\
Mean particle size (nm) & $160-210$ & $175-200$ \\
Polydispersity Index (Pdi) & 0.304 & 0.231 \\
Zeta potential (mV) & $-36.7 \pm 6.10$ & $-31.5 \pm 1.9$ \\
Drug loading (\%) & $33.34 \pm 1.45$ & $16.67 \pm 0.86$ \\
Entrapment efficiency (\%) & $76.24 \pm 1.20$ & $73.75 \pm 1.06$ \\
Drug content (\%) & $96.63 \pm 0.42$ & $94.17 \pm 1.12$ \\
\hline *(n=3 S.D.) & &
\end{tabular}

The results of the drug release profile of Rasagiline mesylate loaded nanoscale solid lipid particle formulations were shown in Fig. 8. The formulations RMSLN-I and RMSLN-II released $92.06 \pm 1.40 \%$ and $89.13 \pm 1.20 \%$, respectively, of their Rasagiline mesylate content at the end of 24 hours.

Each data point in the drug release profile represents the mean of three determinations. The Rasagiline mesylate loaded solid lipid nanoparticles formulations were stored at different conditions includes,

$25^{\circ} \mathrm{C} \pm 2{ }^{\circ} \mathrm{C} / 60 \% \mathrm{RH} \pm 5 \% \mathrm{RH}$, $30^{\circ} \mathrm{C} \pm 2{ }^{\circ} \mathrm{C} / 65 \% \mathrm{RH} \pm 5 \% \mathrm{RH}$ and $40^{\circ} \mathrm{C} \pm 2{ }^{\circ} \mathrm{C} / 75 \% \mathrm{RH} \pm 5 \% \mathrm{RH}$, results were mentioned in the Table 2 . The results shown RMSLNI and RMSLN-II formulations were shown no aggregations or separation over the study period. The particle size of the formulations shows good physical stability of the particle. There was a negligible increase in particle size of RMSLN-II at $40^{\circ} \mathrm{C} \pm 2{ }^{\circ} \mathrm{C} / 75 \% \mathrm{RH} \pm 5 \% \mathrm{RH}$. The magnitude of zeta potential value of RMSLN-I formulation was slightly reduced at $40^{\circ} \mathrm{C} \pm 2^{\circ} \mathrm{C} /$ $75 \%$ RH $\pm 5 \%$ RH.

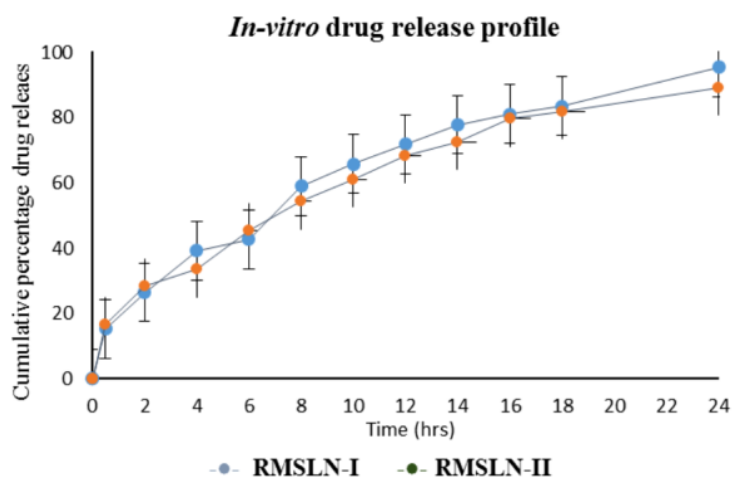

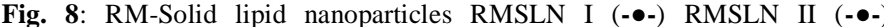
formulations. Each point represents mean \pm standard deviation, $n=3$

No significant changes in the In-vitro drug release of Rasagiline mesylate loaded Nanoscale solid lipid particles. The release of Rasagiline mesylate loaded Nanoscale solid lipid particles results were shown in the table 2. The mechanism of Rasagiline mesylate loaded nanoscale solid lipid particles release was found by incorporating the initial $60 \%$ of the release data in Korsmeyer-Peppas model.

Table 2: Kinetic modeling of RM-loaded nanoscale solid lipid particles.

\begin{tabular}{|c|c|c|c|c|c|}
\hline \multirow{2}{*}{ Formulations } & \multirow{2}{*}{$\begin{array}{c}\text { Zero order } \\
\mathbf{R}^{2}\end{array}$} & \multirow{2}{*}{$\begin{array}{c}\text { First order } \\
\mathbf{R}^{2}\end{array}$} & \multirow{2}{*}{$\frac{\text { Higuchi }}{\mathbf{R}^{2}}$} & \multicolumn{2}{|c|}{ Korosmeyer-Peppas } \\
\hline & & & & $\mathbf{R}^{2}$ & $\mathbf{N}$ \\
\hline RMSLN-I & 0.5970 & 0.9945 & 0.9863 & 0.9876 & 0.490 \\
\hline RMSLN-II & 0.6349 & 0.9925 & 0.9870 & 0.9886 & 0.486 \\
\hline
\end{tabular}

Table3: Stability study of Rasagiline mesylate loaded solid lipid nanoparticles

\begin{tabular}{|c|c|c|c|c|c|}
\hline \multirow{2}{*}{ Formulations } & \multirow{2}{*}{ Conditions } & \multirow{2}{*}{ Evaluation parameters * } & \multicolumn{3}{|c|}{ Observations (Months) } \\
\hline & & & $\mathbf{0}$ & 3 & 6 \\
\hline \multirow{15}{*}{ RMSLN-I } & \multirow{5}{*}{$\begin{array}{l}25^{\circ} \mathrm{C} \pm 2^{\circ} \mathrm{Cl} \\
60 \% \mathrm{RH} \pm 5 \% \mathrm{RH}\end{array}$} & Particle size (nm) & $165-210$ & $165-215$ & $167-220$ \\
\hline & & Polydispersity index (Pdi) & 0.304 & 0.329 & 0.281 \\
\hline & & Zeta potential (mV) & -36.70 & -35.57 & -35.19 \\
\hline & & Entrapment efficiency (\%) & 76.24 & 75.86 & 75.42 \\
\hline & & Drug content $(\%)$ & 96.63 & 96.36 & 96.04 \\
\hline & \multirow{5}{*}{$\begin{array}{l}30^{\circ} \mathrm{C} \pm 2{ }^{\circ} \mathrm{Cl} \\
65 \% \mathrm{RH} \pm 5 \% \mathrm{RH}\end{array}$} & Particle size (nm) & $165-210$ & $170-210$ & $170-220$ \\
\hline & & Polydispersity index (Pdi) & 0.304 & 0.314 & 0.291 \\
\hline & & Zeta potential $(\mathrm{mV})$ & -36.70 & -35.45 & -35.03 \\
\hline & & Entrapment efficiency (\%) & 76.24 & 76.18 & 75.24 \\
\hline & & Drug content $(\%)$ & 96.63 & 96.18 & 96.23 \\
\hline & \multirow{5}{*}{$\begin{array}{l}40^{\circ} \mathrm{C} \pm 2^{\circ} \mathrm{Cl} \\
75 \% \mathrm{RH} \pm 5 \% \mathrm{RH}\end{array}$} & Particle size (nm) & $165-210$ & $170-220$ & $170-230$ \\
\hline & & Polydispersity index (Pdi) & 0.304 & 0.285 & 0.289 \\
\hline & & Zeta potential (mV) & -36.70 & -35.75 & -34.82 \\
\hline & & Entrapment efficiency (\%) & 76.24 & 75.86 & 75.92 \\
\hline & & Drug content $(\%)$ & 96.63 & 96.35 & 95.86 \\
\hline \multirow{15}{*}{ RMSLN-II } & \multirow{5}{*}{$\begin{array}{l}25^{\circ} \mathrm{C} \pm 2^{\circ} \mathrm{Cl} \\
60 \% \mathrm{RH} \pm 5 \% \mathrm{RH}\end{array}$} & Particle size $(\mathrm{nm})$ & $175-200$ & $175-210$ & $175-215$ \\
\hline & & Polydispersity index (Pdi) & 0.231 & 0.218 & 0.204 \\
\hline & & Zeta potential (mV) & -31.5 & -28.35 & -28.51 \\
\hline & & Entrapment efficiency (\%) & 73.75 & 72.48 & 72.21 \\
\hline & & Drug content $(\%)$ & 96.63 & 96.15 & 96.23 \\
\hline & \multirow{5}{*}{$\begin{array}{l}30^{\circ} \mathrm{C} \pm 2{ }^{\circ} \mathrm{Cl} \\
65 \% \mathrm{RH} \pm 5 \% \mathrm{RH}\end{array}$} & Particle size $(\mathrm{nm})$ & $175-200$ & $180-200$ & $175-210$ \\
\hline & & Polydispersity index (Pdi) & 0.231 & 0.224 & 0.207 \\
\hline & & Zeta potential (mV) & -31.50 & -29.43 & -29.61 \\
\hline & & Entrapment efficiency (\%) & 73.75 & 72.15 & 72.34 \\
\hline & & Drug content $(\%)$ & 96.63 & 96.17 & 5.92 \\
\hline & \multirow{5}{*}{$\begin{array}{l}40^{\circ} \mathrm{C} \pm 2^{\circ} \mathrm{Cl} \\
75 \% \mathrm{RH} \pm 5 \% \mathrm{RH}\end{array}$} & Particle size $(\mathrm{nm})$ & $175-200$ & $180-210$ & $180-210$ \\
\hline & & Polydispersity index (Pdi) & 0.231 & 0.208 & 0.214 \\
\hline & & Zeta potential $(\mathrm{mV})$ & -31.5 & -29.54 & -29.47 \\
\hline & & Entrapment efficiency (\%) & 73.75 & 73.42 & 72.94 \\
\hline & & Drug content $(\%)$ & 96.63 & 95.94 & 95.86 \\
\hline
\end{tabular}




\section{DISCUSSION}

Nanoscale solid lipid particles which contain Rasagiline mesylate were fabricated by microemulsion technique and it has been generally the selected technique for the fabrication of targeted drug delivery nanoparticles. Stearic acid was used as biodegradable lipid to encapsulate the drug to facilitate the targeted delivery into brain. Polyethylene-polypropylene glycol has long been used as a steric stabilizer for obtaining nanoscale solid lipid particles. In the presence of polyethylene-polypropylene glycol, following a period of equilibration, stable colloidal particles form, but in the absence of polyethylene-polypropylene glycol the particles are colloidally unstable and rapidly coalesce. Also, it has long been used as a coating agent in nanoparticles, which inhibits the efflux system, especially P-glycoprotein (Pgp) for brain delivery (Kreuter et al., 2002; Ruckmani et al., 2006). Hence, in the current research the polyethylene-polypropylene glycol and polysorbate 80 used to enable an efficient brain delivery of the drugs after iv administration.

The fabricated Rasagiline mesylate loaded nanoscale solid lipid particles, mean particle size range was from 160.12 \pm 1.2 $\mathrm{nm}$ to $210.45 \pm 5.3 \mathrm{~nm}$. Particles below $1 \mu \mathrm{m}$ are an easy accessible in the body, being transported across the blood brain barrier (Viveksarathi and Kannan, 2014). The polydispersity index is a ratio, which determined as a measure of homogeneity. Ideally, should be $<0.3$. RMSLN I \& II particles had polydispersity index 0.304 and 0.231 , respectively and it indicates narrow size distribution and suggested particles are monodispersity. The zeta potential value of RMSLN I \& II formulations value was$36.7 \pm 6.10$ and $-31.5 \pm 1.9 \mathrm{mV}$, respectively. When, the zeta potential values more positive than $30 \mathrm{mV}$ or more negative than $30 \mathrm{mV}$ are electrochemically stable. The loading capacity, which determined the amount of Rasagiline mesylate associated with a unit weight of nanoparticles, was very imperative factor for judging the appropriateness of particular drug-carrier system (Ruckmani et al., 2006). In this study, RMSLN-I and RMSLN-II DL values reached $33.34 \pm 1.45 \%$ and $16.67 \pm 0.86 \% \quad(n=3)$, respectively. The entrapment efficacy results were found greater than $70 \%$ and it was depended upon the drug loading capacity.

Scanning electron microscopy results confirm the morphology of RMSLNs formulated particles were the uniform size with a spherical shape. The transmission electron microscopy results revealed the formulated particles were uniform size and spherical shape with smooth surface. Few particles were aggregates due to their very small dimension with high surface area. The selected area electron diffraction pattern shows the formulated particles are polycrystalline structure. In-vitro Rasagiline mesylate release profile from the formulations RMSLN-I and RMSLN-II, showed the drug release 92.06 $\pm 1.40 \%$ and $89.13 \pm 1.20 \%$, respectively, of their Rasagiline mesylate content at the end of 24 hours. Slight differences in the release profiles of batches RMSLN-I and RMSLN-II was observed, due to the different ratio of drug to stearic acid. Stability studies of Rasagiline mesylate loaded nanoscale solid lipid particles results were shown in Table 2. RMSLN-I and RMSLN-II formulations were shown no aggregations or separation over the 6 months stability study. It was observed that the initial particle size, polydispersity, zeta potential, entrapment efficiency, drug content and In-vitro drug release and the same evaluation parameters of the samples analysed after 0,3 , and 6 months of storage at different conditions as per international conference on harmonisation [(ICH) QIA (R2) guidelines (2003) (27). The results were similar, representing there is no significant variations in the physical as well as chemical characteristics of the RMSLNs formulations. The mechanism of Rasagiline mesylate loaded nanoscale solid lipid particles release from the two formulations were found to be followed Higuchi model, as the plots showed high linearity, with a correlation coefficient $\left(\mathrm{R}^{2}\right)$ value of 0.98 or more. The ' $n$ ' value of Korsmeyer-Peppas model lies below 0.5 and the mechanism controlling the drug release was Fickian diffusion.

\section{CONCLUSION}

The hydrophilic Rasagiline mesylate was successfully encapsulated in stearic acid based solid lipid nanoparticles using microemulsion technique. The particle size distribution was in the nanometre range with polydispersity index and the zeta potential indicates that the solid lipid particles would be stable and the tendency to agglomerate would be miniscule. SEM and TEM studies shown the morphology of RM-SLNs were crystalline and have a spherical shape. Drug loading, entrapment efficiency and drug content results were satisfactory. The release of Rasagiline mesylate from solid lipid nanoparticle was shown efficient prolonged delivery. Furthermore, the Rasagiline mesylate loaded solid lipid nanoparticle formulation were stable for 6 months at different storage conditions and the release data shown there is no significant changes in the In-vitro drug release studies. Hence, the formulated RM loaded SLNs can be used for prolonged drug delivery to improve the bioavailability. However, further studies will focus on increasing the drug loading in nanoscale solid lipid particles.

\section{ACKNOWLEDGMENTS}

The authors are thankful to Department of Pharmacy, Faculty of Engineering and Technology, Annamalai University, Annamalai Nagar, Tamil Nadu, India.

\section{CONFLICTS OF INTEREST}

The authors declare that there are no conflicts of interest.

\section{REFERENCES}

Viveksarathi K, Kannan K. Multi criteria decision making to select the best method for the preparation of solid lipid nanoparticles of rasagiline mesylate using analytic hierarchy process. Journal of Advance Pharmaceutical Technology Research, 2014; 5(3):115-121.

Kersemans K, Van Laeken N, De Vos F. Radiochemistry devoted to the production of monoamine oxidase (MAO-A and MAO-B) 
ligands for brain imaging with positron emission tomography. J Labelled Comp Radiopharm, 2013; 56(3-4):78-88.

Lakshmi Nayak, Claire Henchcliffe. Rasagiline in treatment of Parkinson's disease. Neuropsychiatric Disease and Treatment, 2008; 4(1):11-20.

Marcos Fernandez, Sofia Negroa, Karla Slowing, Ana Fernandez-Carballido, Emilia Barcia. An effective novel delivery strategy of Rasagiline for Parkinson's disease. International Journal of Pharmaceutics, 2011; 419(1-2):271-280.

Moussa BH Youdima, Marta Weinstock. Novel neuroprotective anti-Alzheimer drugs with anti-depressant activity derived from the antiParkinson drug, Rasagiline. Mechanisms of Ageing and Development, 2002; 123(1):1081-1086.

Andreas-Antonios, Roussakis, Paola Piccini, Marios Politis. Clinical utility of DaTscan ${ }^{\mathrm{TM}}$ (123I-Ioflupane Injection) in the diagnosis of Parkinsonian Syndromes. Degenerative Neurological and Neuromuscular Disease. 2013, 3(2):33-39.

Jack J Chen, David M Swope, Khashayar Dashtipour. Comprehensive review of Rasagiline, a second-generation monoamine oxidase inhibitor, for the treatment of Parkinson's disease. Clinical therapeutics, 2007; 29(9):1825-1849.

Haibin LI, Xuexhem DUAN. Nanoparticles for drug delivery to the central nervous system. Nanoscience, 2006; 11(3):207-309.

Mori N, Kurokouchi A, Osonoe K, Saitoh H, Ariga K, Suzuki K. Liposome entrapped phenytoin locally suppresses amygdaloidal epileptogenic focus created by db-CAMP/EDTA in rats. Brain Res, 1995; 703(1-2):184-190.

Kreuter J, Ramge P, Petrov V, Hamm S, Gelperina SE, Engelhardt B. Direct evidence that polysorbate-80-coated poly (butylcyanoacrylate) nanoparticles deliver drugs to the CNS via specific mechanisms requiring prior binding of drug to the nanoparticles. Pharm Res, 2003; 20(3):409-416.

Shuting K, Feng Y, Ying W, Yilin S, Nan Y, Ling Y. The blood-brain barrier penetration and distribution of PEGylated fluoresceindoped magnetic silica nanoparticles in rat brain. Biochem Biophys Res, 2010; 394(4):871-886.

Wolfgang Mehnert, Karsten Mad. Solid lipid nanoparticles Production, characterization and applications. Advanced Drug Delivery Reviews, 2001; 47(1):165-196.

Patricia Severino, Samantha C Pinho, Eliana B. Souto, Maria HA Santana. Polymorphism, crystallinity and hydrophilic-lipophilic balance of stearic acid and stearic acid-capric/caprylic triglyceride matrices for production of stable nanoparticles. Colloids and Surfaces B: Biointerfaces, 2011; 86(2):125-130.

Attama AA, Schicke BC, Muller-Goymann CC. Further characterization of theobroma oil-beeswax admixtures as lipid matrices for improved drug delivery systems. Eur J Pharm Biopharm, 2006; 64(1):294306.

Fundaro A, Cavalli R, Bargoni A, Vighetto D, Zara GP, Gasco MR. Non-stealth and stealth solid lipid nanoparticles (SLN) carrying doxorubicin: pharmacokinetics and tissue distribution after i.v. administration to rats. Pharmacol Res, 2000; 42(3):337-343.

Viveksarathi K, Rajarajan R, Kannan K, Manavalan R. Dosage form design and evaluation of eperisone hydrochloride matrix film coated extended release tablets. Int J Pharm Pharm Sci, 2012; 4(2):575581.

Viveksarathi K, Kannan K. Design and In-vitro evaluation of gastroretentive floating delivery system of rosiglitazone maleate. J Pharm Sci Res, 2013; 5(8):166-170.
Surajit Das, Wai Kiong Ng, Parijat Kanaujia, Sanggu Kim, Reginald BH Tan. Formulation design, preparation and physicochemical characterizations of solid lipid nanoparticles containing a hydrophobic drug: Effects of process variables. Colloids and Surfaces B: Biointerfaces, 2011; 88:483-489.

Viveksarathi K, Kannan K. Compatibility studies of Rasagiline mesylate with selected excipients for an effective solid lipid nanoparticles formulation, Int J Pharm Pharm Sci. 2015a; 7(1):73-80.

Doijad, RC, Manvi FV, Godhwani DM, Joseph R, Deshmukh NV. Formulation and Targeting Efficiency of Cisplatin Engineered Solid Lipid Nanoparticles. Indian Journal of Pharmaceutical Science, 2008; 70(2):203-207.

Barnabas Wilson, Malay Kumar Samanta, Kumaraswamy Santhi, Kokilampal Perumal Sampath Kumar, Nallupillai Paramakrishnan, Bhojraj Suresh. Poly (n-butylcyanoacrylate) nanoparticles coated with polysorbate 80 for the targeted delivery of rivastigmine into the brain to treat Alzheimer's disease. Brain Res, 2008; 1200:159-68.

Viveksarathi K, Kannan K. Effect of the moist-heat sterilization on fabricated nanoscale solid lipid particles containing Rasagiline mesylate. International Journal of Pharmaceutical Investigation, 2015b; $5(2): 1-5$

Marcos Fernandez, Sofia Negroa, Karla Slowing, Ana Fernandez-Carballido, Emilia Barcia. An effective novel delivery strategy of Rasagiline for Parkinson's disease. Int J Pharm, 2011; 419(1-2):271-80.

Guihua Huang, Na Zhang, Xiuli Bi, Mingjin Dou. Solid lipid nanoparticles of temozolomide: Potential reduction of cardial and nephric toxicity, International Journal of Pharmaceutics, 2008; 355:314-320.

Viveksarathi K, Kannan K. Experimental design for the optimization of nanoscale solid lipid particles containing Rasagiline mesylate. Journal of Young Pharmacist, 2015c; 7(4):1-11.

Shengpeng Wang, Tongkai Chen, Ruie Chen, Yangyang Hu, Meiwan Chen, Yitao Wang. Emodin loaded solid lipid nanoparticles: Preparation, characterization and antitumor activity studies. International Journal of Pharmaceutics, 2012; 430:238-246.

ICH Q 1 A (R2). Stability Testing of new Drug Substances and Products. (CPMP/ICH/2736/99). Available from: URL: http://www.emea.eu.int/pdfs/human/ich/273699en.pdf

Kreuter J. Nanoparticulate systems for brain delivery of drugs. Adv Drug Del, 2001; 47:65-81.

Kreuter J, Shamenkov D, Petrov V, Ramge P, Cychutek K, Koch-Brandt C, et al. Apolipoprotein-mediated transport of nanoparticlebound drugs across the blood-brain barrier. Drug Target, 2002; 10(4):317325 .

Ruckmani K, Sivakumar M, Ganeshkumar PA. Methotrexate loaded solid lipid nanoparticles (SLN) for effective treatment of carcinoma, J Nanosci Nanotechnol, 2006; 6(9-10):1-5.

Viveksarathi K, Kannan K. Kinetic modeling of rasagiline mesylate from nanoscale solid lipid particles, International Journal of Pharmacy and Pharmaceutical Sciences, 2015; 7(11): 300-305.

\section{How to cite this article:}

Kunasekaran V, Krishnamoorthy K. Formulation and Evaluation of Nanoscale Solid Lipid Particles Containing a Hydrophilic Drug Rasagiline Mesylate. J App Pharm Sci, 2016; 6 (05): 044-050. 